\title{
WEAK GALERKIN FINITE ELEMENT METHODS FOR PARABOLIC EQUATIONS
}

\author{
QIAOLUAN H. LI AND JUNPING WANG
}

\begin{abstract}
A newly developed weak Galerkin method is proposed to solve parabolic equations. This method allows the usage of totally discontinuous functions in approximation space and preserves the energy conservation law. Both continuous and discontinuous time weak Galerkin finite element schemes are developed and analyzed. Optimal order error estimates in both $H^{1}$ and $L^{2}$ norms are established. Numerical tests are performed and reported.
\end{abstract}

\section{INTRODUCTION}

In this paper, we consider the initial-boundary value problem

$$
\begin{aligned}
& u_{t}-\nabla \cdot(a \nabla u)=f \quad \text { in } \Omega, \quad t \in J, \\
& u=g \quad \text { on } \partial \Omega, \quad t \in J, \\
& u(\cdot, 0)=\psi \text { in } \Omega,
\end{aligned}
$$

where $\Omega$ is a polygonal or polyhedral domain in $\mathbb{R}^{d}(d=2,3)$ with Lipschitz-continuous boundary $\partial \Omega, J=(0, \bar{t}]$, and $a=\left(a_{i j}(x, t)\right)_{d \times d} \in\left[L^{\infty}(\Omega \times \bar{J})\right]^{d^{2}}$ is a symmetric matrix-valued function satisfying the following property: there exists a constant $\alpha>0$ such that

$$
\alpha \xi^{T} \xi \leq \xi^{T} a \xi, \quad \forall \xi \in \mathbb{R}^{d}
$$

For simplicity, we shall concentrate on two-dimensional problems only (i.e., $d=2$ ).

For any nonnegative integer $m$, let $H^{m}(\Omega)$ be the standard Sobolov space, which is the collection of all real-valued functions defined on $\Omega$ with square integrable derivatives up to order $m$ with semi-norm

$$
|\psi|_{s, \Omega} \equiv\left\{\sum_{|\alpha|=s} \int_{\Omega}\left|\partial^{\alpha} \psi\right|^{2} d x\right\}^{\frac{1}{2}},
$$

and norm

$$
\|\psi\|_{m, \Omega} \equiv\left(\sum_{j=0}^{m}|\psi|_{j, \Omega}^{2}\right)^{\frac{1}{2}} .
$$

For simplicity, we use $\|\cdot\|$ for the $L^{2}$ norm.

The parabolic problem can be written in a variational form as follows

$$
\begin{aligned}
\left(u_{t}, v\right)+(a \nabla u, \nabla v) & =(f, v) \quad \forall v \in H_{0}^{1}(\Omega), t \in J, \\
u(\cdot, 0) & =\psi,
\end{aligned}
$$

where $u$ is called a weak solution if $u \in L^{2}\left(0, t ; H^{1}(\Omega)\right)$ and $u_{t} \in L^{2}\left(0, t ; H^{-1}(\Omega)\right)$, and if $u=g$ on $\partial \Omega$.

2010 Mathematics Subject Classification. Primary 65N15, 65N30, 76D07; Secondary, 35B45, 35J50.

Key words and phrases. weak Galerkin methods, finite element methods, parabolic equations.

The research of Wang was supported by the NSF IR/D program, while working at the National Science Foundation. However, any opinion, finding, and conclusions or recommendations expressed in this material are those of the author and do not necessarily reflect the views of the National Science Foundation. 
Parabolic problems have been treated by various numerical methods. For finite element methods, we refer to [1] and 2]. Discontinuous Galerkin finite element methods were studied in [3] and [4]. In [5] and [6], finite volume methods were presented, which were based on the integral conservation law rather than the differential equation. The integral conservation law was then enforced for small control volumes defined by the computational mesh.

The goal of this paper is to consider a newly developed weak Galerkin (WG) finite element method for parabolic equation which is based on the definition of a discrete weak gradient operator proposed in [7. In this numerical method, the analysis can be done by using the framework of Galerkin methods, and totally discontinuous functions are allowed to be used as the approximation space. Furthermore, the approximation results also satisfy the energy conservation law.

The rest of this paper is organized as follows. In Section 2, we introduce some notation and establish a continuous time and discontinuous time weak Galerkin finite element scheme for the parabolic initial boundary-value problem (11). In Section 3, we prove the energy conservation law of the weak Galerkin approximation. Optimal order error estimates in both $H^{1}$ and $L^{2}$ norms are proved in Section 4. The paper is concluded with some numerical experiments to illustrate the theoretical analysis in Section 5.

\section{The Weak Galerkin Method}

In this section we design a continuous time and a discontinuous time weak Galerkin finite element scheme for the initial-boundary value problem (11). We consider the space of discrete weak functions and the discrete weak operator introduced in [7]. Let $\mathcal{T}_{h}$ be a quasiuniform family of triangulations of a plane domain $\Omega$ and $T$ be each triangle element with $\partial T$ as its boundary. For each $T \in \mathcal{T}_{h}$, let $P_{j}(T)$ be the set of polynomials on $T$ with degree no more than $j$, and $P_{l}(\partial T)$ be the set of polynomials on $\partial T$ with degree no more than $l$, respectively. Let $\hat{P}_{j}(T)$ be the set of homogeneous polynomials on $T$ with degree $j$. The weak finite element space $S_{h}(j, l)$ is defined by

$$
S_{h}(j, l):=\left\{v=\left\{v_{0}, v_{b}\right\}: v_{0} \in P_{j}(T), v_{b} \in P_{l}(e) \text { for all edge } e \subset \partial T, T \in \mathcal{T}_{h}\right\} .
$$

Denote by $S_{h}^{0}(j, l)$ the subspace of $S_{h}(j, l)$ with vanishing boundary value on $\partial \Omega$; i.e.,

$$
S_{h}^{0}(j, l):=\left\{v=\left\{v_{0}, v_{b}\right\} \in S_{h}(j, l),\left.v_{b}\right|_{\partial T \cap \partial \Omega}=0 \text { for all } T \in \mathcal{T}_{h}\right\} .
$$

Let $\sum_{h}=\left\{\mathbf{q} \in\left[L^{2}(\Omega)\right]^{2}:\left.\mathbf{q}\right|_{T} \in V(T, r)\right.$ for all $\left.T \in \mathcal{T}_{h}\right\}$, where $V(T, r)$ is a subspace of the set of vector-valued polynomials of degree no more than $r$ on $T$. For each $v=\left\{v_{0}, v_{b}\right\} \in S_{h}(j, l)$, the discrete weak gradient $\nabla_{d} v \in \sum_{h}$ of $v$ on each element $T$ is given by the following equation:

$$
\int_{T} \nabla_{d} v \cdot \mathbf{q} d T=-\int_{T} v_{0} \nabla \cdot \mathbf{q} d T+\int_{\partial T} v_{b} \mathbf{q} \cdot \mathbf{n} d s, \quad \forall \mathbf{q} \in V(T, r),
$$

where $\mathbf{n}$ is the outward normal direction of $\partial T$. It is easy to see that this discrete weak gradient is well-defined.

To investigate the approximation properties of the discrete weak spaces $S_{h}(j, l)$ and $\sum_{h}$, we use three projections in this paper. The first two are local projections defined on each triangle $T$ : one is $Q_{h} u=\left\{Q_{0} u, Q_{b} u\right\}$, the $L^{2}$ projection of $H^{1}(T)$ onto $P_{j}(T) \times P_{l}(\partial T)$ and another is $\mathbb{Q}_{h}$, the $L^{2}$ projection of $\left[L^{2}(T)\right]^{2}$ onto $V(T, r)$. The third projection $\Pi_{h}$ is assumed to exist and satisfy the following property: For $\mathbf{q} \in H(\operatorname{div}, \Omega)$ with mildly added regularity, $\Pi_{h} \mathbf{q} \in H($ div,$\Omega)$ such that $\Pi_{h} \mathbf{q} \in V(T, r)$ on each $T \in \mathcal{T}_{h}$, and

$$
\left(\nabla \cdot \mathbf{q}, v_{0}\right)_{T}=\left(\nabla \cdot \Pi_{h} \mathbf{q}, v_{0}\right)_{T}, \quad \forall v_{0} \in P_{j}(T) .
$$

It is easy to see the following two useful identities:

$$
\nabla_{d}\left(Q_{h} w\right)=\mathbb{Q}_{h}(\nabla w), \quad \forall w \in H^{1}(T),
$$


and for any $\mathbf{q} \in H(\operatorname{div}, \Omega)$

$$
\sum_{T \in \mathcal{T}_{h}}\left(-\nabla \cdot \mathbf{q}, v_{0}\right)_{T}=\sum_{T \in \mathcal{T}_{h}}\left(\Pi_{h} \mathbf{q}, \nabla_{d} v\right)_{T}, \quad \forall v=\left\{v_{0}, v_{b}\right\} \in S_{h}^{0}(j, l) .
$$

The discrete weak spaces $S_{h}(j, l)$ and $\sum_{h}$ need to possess some good approximation properties in order to provide an acceptable finite element scheme. In [7, the following two criteria were given as a general guideline for their construction:

P1: For any $v \in S_{h}(j, l)$, if $\nabla_{d} v=0$ on $T$, then one must have $v \equiv$ constant on $T$; i.e., $v_{0}=v_{b}=$ constant on $T$.

P2: For any $u \in H_{0}^{1}(\Omega) \cap H^{m+1}(\Omega)$, where $0 \leq m \leq j+1$, the discrete weak gradient of the $L^{2}$ projection $Q_{h} u$ of $u$ in the discrete weak space $S_{h}(j, l)$ provides a good approximation of $\nabla u$; i.e., $\left\|\nabla_{d}\left(Q_{h} u\right)-\nabla u\right\| \leq C h^{m}\|u\|_{m+1}$ holds true.

Examples of $S_{h}(j, l)$ and $\sum_{h}$ satisfying the conditions $\mathbf{P 1}$ and $\mathbf{P 2}$ can be found in [7]. For example, with $V(T, r=j+1)=\left[P_{j}(T)\right]^{2}+\hat{P}_{j}(T) \mathbf{x}$ being the usual Raviart-Thomas element [8] of order $j$, one may take equal-order elements of order $j$ for $S_{h}(j, l)$ in the interior and the boundary of each element $T$. The key of using the Raviart-Thomas element for $V(T, r)$ is to ensure the condition P1. The condition P2 was satisfied by the commutative property (4) which has been established in [7]. It was shown later in [9, 10, that the condition $\mathbf{P 1}$ can be circumvented by a suitable stabilization term. Consequently, the selection of $V(T, r)$ and $S_{h}(j, l)$ becomes very flexible and robust in practical computation. It even allows the use of finite elements of arbitrary shape.

The main idea of the weak Galerkin method is to use the discrete weak space $S_{h}(j, l)$ as testing and trial spaces and replace the classical gradient operator by the weak gradient operator $\nabla_{d}$ in (2).

First, we pose the continuous time weak Galerkin finite element method, based on the weak Galerkin operator (3) and weak formulation (2), which is to find $u_{h}(t)=\left\{u_{0}(\cdot, t), u_{b}(\cdot, t)\right\}$, belonging to $S_{h}(j, l)$ for $t \geq 0$, satisfying $u_{b}=Q_{b} g$ on $\partial \Omega, t>0$ and $u_{h}(0)=Q_{h} \psi$ in $\Omega$, and the following equation

$$
\left(\left(u_{h}\right)_{t}, v_{0}\right)+a\left(u_{h}, v\right)=\left(f, v_{0}\right) \quad \forall v=\left\{v_{0}, v_{b}\right\} \in S_{h}^{0}(j, l), t>0
$$

where $a(\cdot, \cdot)$ is the weak bilinear form defined by

$$
a(w, v)=\left(a \nabla_{d} w, \nabla_{d} v\right),
$$

which is assumed to be bounded and coercive, i.e., for constant $\alpha, \beta, \gamma>0$

$$
\begin{gathered}
|a(u, v)| \leq \beta\left\|\nabla_{d} u\right\|\left\|\nabla_{d} v\right\|, \\
a(u, u) \geq \alpha\left\|\nabla_{d} u\right\|^{2},
\end{gathered}
$$

and that

$$
\left|\left(a_{t} \nabla_{d} u, \nabla_{d} v\right)\right| \leq \gamma\left\|\nabla_{d} u\right\|\left\|\nabla_{d} v\right\| .
$$

We now turn our attention to some discrete time Weak Galerkin procedures. We introduce a time step $k$ and the time levels $t=t_{n}=n k$, where $n$ is a nonnegative integer, and denote by $U^{n}=U_{h}^{n} \in S_{h}(j, l)$ the approximation of $u\left(t_{n}\right)$ to be determined. The backward Euler Weak Galerkin method is defined by replacing the time derivative in (6) by a backward difference quotient, or, if $\bar{\partial} U^{n}=\left(U^{n}-U^{n-1}\right) / k$,

$$
\left(\bar{\partial} U^{n}, v_{0}\right)+a\left(U^{n}, v\right)=\left(f\left(t_{n}\right), v_{0}\right) \quad \forall v=\left\{v_{0}, v_{b}\right\} \in S_{h}^{0}(j, l), n \geq 1, U^{0}=Q_{h} \psi,
$$

i.e.,

$$
\left(U^{n}, v_{0}\right)+k a\left(U^{n}, v\right)=\left(U^{n-1}+k f\left(t_{n}\right), v_{0}\right), \quad \forall v=\left\{v_{0}, v_{b}\right\} \in S_{h}^{0}(j, l)
$$




\section{Energy Conservation of Weak Galerkin}

This section investigates the energy conservation property of the weak Galerkin finite element approximation $u_{h}$. The increase in internal energy in a small spatial region of the material $K$, i.e., control volume, over the time period $[t-\Delta t, t+\Delta t]$ is given by

$$
\int_{K} u(x, t+\Delta t) d x-\int_{K} u(x, t-\Delta t) d x=\int_{t-\Delta t}^{t+\Delta t} \int_{K} u_{t} d x d t .
$$

Suppose that a body obeys the heat equation and, in addition, generates its own heat per unit volume at a rate given by a known function $f$ varying in space and time, the change in internal energy in $K$ is accounted for by the flux of heat across the boundaries together with the source energy. By Fourier's law, this is

$$
-\int_{t-\Delta t}^{t+\Delta t} \int_{\partial K} q \cdot \mathbf{n} d s d t+\int_{t-\Delta t}^{t+\Delta t} \int_{K} f d x d t
$$

where $q=-a \nabla u$ is the flow rate of heat energy. The solution $u$ of the problem (11) yields the following integral form of energy conservation:

$$
\int_{t-\Delta t}^{t+\Delta t} \int_{K} u_{t} d x d t+\int_{t-\Delta t}^{t+\Delta t} \int_{\partial K} q \cdot \mathbf{n} d s d t=\int_{t-\Delta t}^{t+\Delta t} \int_{K} f d x d t
$$

where the Green's formula was used. We claim that the numerical approximation from the weak Galerkin finitel element method for (11) retains the energy conservation property (8).

In (6), we chose a test function $v=\left\{v_{0}, v_{b}=0\right\}$ so that $v_{0}=1$ on $K$ and $v_{0}=0$ elsewhere. After integrating over the time period, we have

$$
\int_{t-\Delta t}^{t+\Delta t} \int_{K} u_{t} d x d t+\int_{t-\Delta t}^{t+\Delta t} \int_{K} a \nabla_{d} u \nabla_{d} v d x d t=\int_{t-\Delta t}^{t+\Delta t} \int_{K} f d x d t
$$

Using the definition of operator $\mathbb{Q}_{h}$ and of the weak gradient $\nabla_{d}$ in (3), we arrive at

$$
\int_{K} a \nabla_{d} u \nabla_{d} v d x=\int_{K} \mathbb{Q}_{h}\left(a \nabla_{d} u_{h}\right) \cdot \nabla_{d} v d x=-\int_{K} \nabla \cdot \mathbb{Q}_{h}\left(a \nabla_{d} u_{h}\right) d x=-\int_{\partial K} \mathbb{Q}_{h}\left(a \nabla_{d} u_{h}\right) \cdot \mathbf{n} d s .
$$

Then by substituting in (9), we have the energy conservation property

$$
\int_{t-\Delta t}^{t+\Delta t} \int_{K} u_{t} d x d t+\int_{t-\Delta t}^{t+\Delta t} \int_{\partial K}-\mathbb{Q}_{h}\left(a \nabla_{d} u_{h}\right) \cdot \mathbf{n} d s d t=\int_{t-\Delta t}^{t+\Delta t} \int_{K} f d x d t,
$$

which provides a numerical flux given by

$$
q_{h} \cdot \mathbf{n}=-\mathbb{Q}_{h}\left(a \nabla_{d} u_{h}\right) \cdot \mathbf{n} .
$$

The numerical flux $q_{h} \cdot n$ is continuous across the edge of each element $T$, which can be verified by a selection of the test function $v=\left\{v_{0}, v_{b}\right\}$ so that $v_{0} \equiv 0$ and $v_{b}$ arbitrary.

\section{ERror AnALYsis}

In this section, we derive some error estimates for both continuous and discrete time weak Galerkin finite element methods. The difference between the weak Galerkin finite element approximation $u_{h}$ and the $L^{2}$ projection $Q_{h} u$ of the exact solution $u$ is measured. We first state a result concerning an approximation property as follows.

Lemma 4.1. For $u \in H^{1+r}(\Omega)$ with $r>0$, we have

$$
\left\|\Pi_{h}(a \nabla u)-a \mathbb{Q}_{h}(\nabla u)\right\| \leq C h^{r}\|u\|_{1+r} .
$$


Proof. Since from (4) we have $\mathbb{Q}_{h}(\nabla u)=\nabla_{d}\left(Q_{h} u\right)$, then

$$
\left\|\Pi_{h}(a \nabla u)-a \mathbb{Q}_{h}(\nabla u)\right\|=\left\|\Pi_{h}(a \nabla u)-a \nabla_{d}\left(Q_{h} u\right)\right\| .
$$

Using the triangle inequality, the definition of $\Pi_{h}$ and the second condition P2 on the discrete weak space $S_{h}(j, l)$, we have

$$
\left\|\Pi_{h}(a \nabla u)-a \nabla_{d}\left(Q_{h} u\right)\right\| \leq\left\|\Pi_{h}(a \nabla u)-a \nabla u\right\|+\left\|a \nabla u-a \nabla_{d}\left(Q_{h} u\right)\right\| \leq C h^{r}\|u\|_{1+r} .
$$

4.1. Continuous Time Weak Galerkin Finite Element Method. Our aim is to prove the following estimate for the error for the semidiscrete solution.

Theorem 4.1. Let $u \in H^{1+r}(\Omega)$ and $u_{h}$ be the solutions of (1) and (6), respectively. Denote by $e:=u_{h}-Q_{h} u$ the difference between the weak Galerkin approximation and the $L^{2}$ projection of the exact solution $u$. Then there exists a constant $C$ such that

$$
\|e(\cdot, t)\|^{2}+\int_{0}^{t} \alpha\left\|\nabla_{d} e\right\|^{2} d s \leq\|e(\cdot, 0)\|^{2}+C h^{2 r} \int_{0}^{t}\|u\|_{1+r}^{2} d s,
$$

and

$$
\begin{aligned}
& \int_{0}^{t}\left\|e_{t}\right\|^{2} d s+\frac{\alpha}{4}\left\|\nabla_{d} e(\cdot, t)\right\|^{2}+\left(1+\frac{\gamma}{2 \alpha}\right)\|e\|^{2} \\
\leq & \alpha\left\|\nabla_{d} e(\cdot, 0)\right\|^{2}+\left(1+\frac{\gamma}{2 \alpha}\right)\|e(\cdot, 0)\|^{2} \\
& +C h^{2 r}\left(\|u(\cdot, 0)\|_{1+r}^{2}+\|u\|_{1+r}^{2}+\int_{0}^{t}\|u\|_{1+r}^{2} d s+\int_{0}^{t}\left\|u_{t}\right\|_{1+r}^{2} d s\right) .
\end{aligned}
$$

Proof. Let $v=\left\{v_{0}, v_{b}\right\} \in S_{h}^{0}(j, l)$ be the testing function. By testing (1) against $v_{0}$, together with $\mathbb{Q}_{h}(\nabla u)=\nabla_{d}\left(Q_{h} u\right)$ for $u \in H^{1}$ and $\left(Q_{0} u_{t}, v_{0}\right)=\left(u_{t}, v_{0}\right)$, we obtain

$$
\begin{aligned}
\left(f, v_{0}\right) & =\left(u_{t}, v_{0}\right)+\sum_{T \in \mathcal{T}_{h}}\left(-\nabla \cdot(a \nabla u), v_{0}\right)_{T} \\
& =\left(u_{t}, v_{0}\right)+\left(\Pi_{h}(a \nabla u), \nabla_{d} v\right) \\
& =\left(Q_{h} u_{t}, v_{0}\right)+\left(\Pi_{h}(a \nabla u)-a \mathbb{Q}_{h}(\nabla u), \nabla_{d} v\right)+\left(a \nabla_{d}\left(Q_{h} u\right), \nabla_{d} v\right)
\end{aligned}
$$

Since the numerical solution also satisfies the heat equation, we have

$$
\left(f, v_{0}\right)=\left(\left(u_{h}\right)_{t}, v_{0}\right)+a\left(u_{h}, v\right) .
$$

Combining the above two equations we obtain

$$
\left(\left(u_{h}-Q_{h} u\right)_{t}, v_{0}\right)+a\left(u_{h}-Q_{h} u, v\right)=\left(\Pi_{h}(a \nabla u)-a \mathbb{Q}_{h}(\nabla u), \nabla_{d} v\right),
$$

which shall be called the error equation for the WG for the heat equation.

Let $e=u_{h}-Q_{h} u$ be the error. Use $v=e$ in the error equation, we obtain

$$
\left(e_{t}, e\right)+a(e, e)=\left(\Pi_{h}(a \nabla u)-a \mathbb{Q}_{h}(\nabla u), \nabla_{d} e\right) .
$$

By Cauchy-Schwarz inequality and the coercivity of the bilinear form, we have

$$
\frac{1}{2} \frac{d}{d t}\|e\|^{2}+\alpha\left\|\nabla_{d} e\right\|^{2} \leq \frac{1}{2 \alpha}\left\|\Pi_{h}(a \nabla u)-a \mathbb{Q}_{h}(\nabla u)\right\|^{2}+\frac{\alpha}{2}\left\|\nabla_{d} e\right\|^{2} .
$$

It follows that

$$
\frac{d}{d t}\|e\|^{2}+\alpha\left\|\nabla_{d} e\right\|^{2} \leq \frac{1}{\alpha}_{5}\left\|\Pi_{h}(a \nabla u)-a \mathbb{Q}_{h}(\nabla u)\right\|^{2}
$$


and hence, after integration,

$$
\|e\|^{2}+\int_{0}^{t} \alpha\left\|\nabla_{d} e\right\|^{2} d s \leq\|e(\cdot, 0)\|^{2}+\frac{1}{\alpha} \int_{0}^{t}\left\|\Pi_{h}(a \nabla u)-a \mathbb{Q}_{h}(\nabla u)\right\|^{2} d t .
$$

Then by Lemma 4.1, we have the assertion.

In order to estimate $\nabla_{d} e$, we use the error equation with $v=\left(u_{h}-Q_{h} u\right)_{t}=e_{t}$. We obtain

$$
\begin{aligned}
\left(e_{t}, e_{t}\right)+a\left(e, e_{t}\right)= & \left(\Pi_{h}(a \nabla u)-a \mathbb{Q}_{h}(\nabla u), \nabla_{d} e_{t}\right) \\
= & \frac{d}{d t}\left(\Pi_{h}(a \nabla u)-a \mathbb{Q}_{h}(\nabla u), \nabla_{d} e\right) \\
& -\left(\Pi_{h}\left(a \nabla u_{t}\right)-a \mathbb{Q}_{h}\left(\nabla u_{t}\right), \nabla_{d} e\right) \\
& -\left(\Pi_{h}\left(a_{t} \nabla u\right)-a_{t} \mathbb{Q}_{h}(\nabla u), \nabla_{d} e\right) .
\end{aligned}
$$

By the Cauchy-Schwarz inequality, this shows that

$$
\begin{aligned}
\left\|e_{t}\right\|^{2}+\frac{1}{2}\left(\frac{d}{d t} a(e, e)-\left(a_{t} \nabla_{d} e, \nabla_{d} e\right)\right) & \leq \frac{d}{d t}\left(\Pi_{h}(a \nabla u)-a \mathbb{Q}_{h}(\nabla u), \nabla_{d} e\right) \\
& +\frac{1}{2 \alpha}\left\|\Pi_{h}\left(a \nabla u_{t}\right)-a \mathbb{Q}_{h}\left(\nabla u_{t}\right)\right\|^{2}+\frac{\alpha}{2}\left\|\nabla_{d} e\right\|^{2} \\
& +\frac{1}{2 \alpha}\left\|\Pi_{h}\left(a_{t} \nabla u\right)-a_{t} \mathbb{Q}_{h}(\nabla u)\right\|^{2}+\frac{\alpha}{2}\left\|\nabla_{d} e\right\|^{2},
\end{aligned}
$$

i.e.,

$$
\begin{aligned}
\left\|e_{t}\right\|^{2}+\frac{1}{2} \frac{d}{d t} a(e, e) & \leq \frac{1}{2}\left(a_{t} \nabla_{d} e, \nabla_{d} e\right)+\frac{d}{d t}\left(\Pi_{h}(a \nabla u)-a \mathbb{Q}_{h}(\nabla u), \nabla_{d} e\right) \\
& +\frac{1}{2 \alpha}\left\|\Pi_{h}\left(a \nabla u_{t}\right)-a \mathbb{Q}_{h}\left(\nabla u_{t}\right)\right\|^{2}+\frac{\alpha}{2}\left\|\nabla_{d} e\right\|^{2} \\
& +\frac{1}{2 \alpha}\left\|\Pi_{h}\left(a_{t} \nabla u\right)-a_{t} \mathbb{Q}_{h}(\nabla u)\right\|^{2}+\frac{\alpha}{2}\left\|\nabla_{d} e\right\|^{2} .
\end{aligned}
$$

Thus, integrating with respect to $t$ and together with the coercivity and boundedness yields

$$
\begin{aligned}
& \int_{0}^{t}\left\|e_{t}\right\|^{2} d s+\frac{\alpha}{2}\left\|\nabla_{d} e(\cdot, t)\right\|^{2} \\
\leq & \frac{\beta}{2}\left\|\nabla_{d} e(\cdot, 0)\right\|^{2}+\left(\Pi_{h}\left(a \nabla u(\cdot, t)-a \mathbb{Q}_{h}(\nabla u(\cdot, t)), \nabla_{d} e(\cdot, t)\right)\right. \\
- & \left(\Pi_{h}\left(a \nabla u(\cdot, 0)-a \mathbb{Q}_{h}(\nabla u(\cdot, 0)), \nabla_{d} e(\cdot, 0)\right)+\frac{1}{2 \alpha} \int_{0}^{t}\left\|\Pi_{h}\left(a \nabla u_{t}\right)-a \mathbb{Q}_{h}\left(\nabla u_{t}\right)\right\|^{2} d s\right. \\
+ & \frac{1}{2 \alpha} \int_{0}^{t}\left\|\Pi_{h}\left(a_{t} \nabla u\right)-a_{t} \mathbb{Q}_{h}(\nabla u)\right\|^{2} d s+\left(\alpha+\frac{\gamma}{2}\right) \int_{0}^{t}\left\|\nabla_{d} e\right\|^{2} d s .
\end{aligned}
$$

By adding $(\alpha+\gamma / 2) / \alpha=1+\frac{\gamma}{2 \alpha}$ times inequality (11) to the above inequality, we arrive at

$$
\begin{aligned}
& \int_{0}^{t}\left\|e_{t}\right\|^{2} d s+\frac{\alpha}{2}\left\|\nabla_{d} e(\cdot, t)\right\|^{2}+\left(1+\frac{\gamma}{2 \alpha}\right)\|e\|^{2} \\
\leq & \frac{\beta}{2}\left\|\nabla_{d} e(\cdot, 0)\right\|^{2}+\left(1+\frac{\gamma}{2 \alpha}\right)\|e(\cdot, 0)\|^{2} \\
+ & \left(\Pi_{h}(a \nabla u(\cdot, t))-a \mathbb{Q}_{h}(\nabla u(\cdot, t)), \nabla_{d} e(\cdot, t)\right)-\left(\Pi_{h}(a \nabla u(\cdot, 0))-a \mathbb{Q}_{h}(\nabla u(\cdot, 0)), \nabla_{d} e(\cdot, 0)\right) \\
+ & \frac{1}{2 \alpha} \int_{0}^{t}\left\|\Pi_{h}\left(a \nabla u_{t}\right)-a \mathbb{Q}_{h}\left(\nabla u_{t}\right)\right\|^{2} d s+\frac{1}{2 \alpha} \int_{0}^{t}\left\|\Pi_{h}\left(a_{t} \nabla u\right)-a_{t} \mathbb{Q}_{h}(\nabla u)\right\|^{2} d s \\
+ & \left(\frac{1}{\alpha}+\frac{\gamma}{2 \alpha^{2}}\right) \int_{0}^{t}\left\|\Pi_{h}(a \nabla u)-a \mathbb{Q}_{h}(\nabla u)\right\|^{2} d t .
\end{aligned}
$$


Next, we use the Cauchy-Schwarz inequality to obtain

$$
\begin{aligned}
& \int_{0}^{t}\left\|e_{t}\right\|^{2} d s+\frac{\alpha}{4}\left\|\nabla_{d} e(\cdot, t)\right\|^{2}+\left(1+\frac{\gamma}{2 \alpha}\right)\|e\|^{2} \\
\leq & \beta\left\|\nabla_{d} e(\cdot, 0)\right\|^{2}+\left(1+\frac{\gamma}{2 \alpha}\right)\|e(\cdot, 0)\|^{2} \\
+ & \frac{1}{\alpha}\left\|\Pi_{h}(a \nabla u(\cdot, t))-a \mathbb{Q}_{h}(\nabla u(\cdot, t))\right\|^{2}+\frac{1}{2 \alpha}\left\|\Pi_{h}(a \nabla u(\cdot, 0))-a \mathbb{Q}_{h}(\nabla u(\cdot, 0))\right\|^{2} \\
+ & \frac{1}{2 \alpha} \int_{0}^{t}\left\|\Pi_{h}\left(a \nabla u_{t}\right)-a \mathbb{Q}_{h}\left(\nabla u_{t}\right)\right\|^{2} d s+\frac{1}{2 \alpha} \int_{0}^{t}\left\|\Pi_{h}\left(a_{t} \nabla u\right)-a_{t} \mathbb{Q}_{h}(\nabla u)\right\|^{2} d s \\
+ & \left(\frac{1}{\alpha}+\frac{\gamma}{2 \alpha^{2}}\right) \int_{0}^{t}\left\|\Pi_{h}(a \nabla u)-a \mathbb{Q}_{h}(\nabla u)\right\|^{2} d t .
\end{aligned}
$$

Then by Lemma 4.1, the proof is completed.

4.2. Discrete Time Weak Galerkin Finite Element Method. We begin with the following lemma which provides a Poincaré-type inequality with the weak gradient operator.

Lemma 4.2. Assume that $\phi=\left\{\phi_{0}, \phi_{b}\right\} \in S_{h}^{0}(j, j)$, then there exists a constant $C$ such that

$$
\|\phi\| \leq C\left\|\nabla_{d} \phi\right\|
$$

where $\nabla_{d} \phi \in V(T, r=j+1)=\left[P_{j}(T)\right]^{2}+\hat{P}_{j}(T) \mathbf{x}$.

Proof. Let $\bar{\phi}_{0}$ be a piecewise constant function with the cell average of $\phi$ on each element $T$. Let $\phi_{I} \in H_{0}^{1}(\Omega)$ be a continuous piecewise polynomial with vanishing boundary value lifted from $\phi$ as follows. Let $G_{j}(T)$ be the set of all Lagrangian nodal points for $P_{j+1}(T)$. At all internal Lagrangian nodal points $x_{i} \in G_{j}(T)$, we set $\phi_{I}\left(x_{i}\right)=\bar{\phi}_{0}$. At boundary Lagrangian points $x_{i} \in G_{j}(T) \cap \partial T$, we let $\phi_{I}\left(x_{i}\right)$ be the trace of $\bar{\phi}_{0}$ from either side of the boundary. At global Lagrangian points $x_{i} \in \partial T \cap \partial \Omega$, we set $\phi_{I}\left(x_{i}\right)=0$. Let $\llbracket \phi_{0} \rrbracket_{e}$ denote the jump of $\phi_{0}$ on the edge $e$; i.e.,

$$
\llbracket \phi_{0} \rrbracket_{e}=\left.\phi_{0}\right|_{T_{1}}-\left.\phi_{0}\right|_{T_{2}}=\left(\left.\phi_{0}\right|_{T_{1}}-\phi_{b}\right)-\left(\left.\phi_{0}\right|_{T_{2}}-\phi_{b}\right) .
$$

By the classical Poincaré inequality for $\phi_{I}$, we have

$$
\begin{aligned}
\|\phi\| & \leq\left\|\phi-\phi_{I}\right\|+\left\|\phi_{I}\right\| \\
& \leq\left(\sum_{T}\left\|\phi-\phi_{I}\right\|_{T}^{2}\right)^{\frac{1}{2}}+C\left\|\nabla \phi_{I}\right\| .
\end{aligned}
$$

From Lemma 4.3 in [11, we have

$$
\left\|\phi-\phi_{I}\right\|_{T}^{2} \leq \sum_{T^{\prime} \in \mathcal{T}(T)} h_{T^{\prime}}^{2}\left\|\nabla \phi_{0}\right\|_{T^{\prime}}^{2}+\sum_{e \in \varepsilon(T)} h_{e}\left\|\llbracket \phi_{0} \rrbracket\right\|_{e}^{2}, \quad \forall T \in \mathcal{T}_{h},
$$

where $\mathcal{T}(T)$ denotes the set of all triangles in $\mathcal{T}$ having a nonempty intersect with $T$, including $T$ itself, and $\varepsilon(T)$ denotes the set of all edges having a nonempty intersection with $T$. Note the elementary fact that

$$
\left\|\nabla \phi_{I}\right\|^{2} \leq C \sum_{T}\left|\phi_{I}\left(x_{i}\right)-\phi_{I}\left(x_{k}\right)\right|^{2}
$$

where $x_{i}$ and $x_{k}$ run through all the Lagrangian nodal points on $T$. By construction, $\phi_{I}\left(x_{i}\right)$ is the cell average of $\phi_{0}$ on either the element $T$ or an adjacent element $T_{*}$ which shares with $T$ a common edge or a vertex point. Thus, $\phi_{I}\left(x_{i}\right)-\phi_{I}\left(x_{k}\right)$ is either zero or the difference of the cell average of 
$\phi_{0}$ on two adjacent elements $T$ and $T_{*}$. For the later case, assume that $x_{e}$ is a point shared by $T$ and $T_{*}$. It is not hard to see that

$$
\left|\bar{\phi}_{0}\right|_{T}-\left.\phi_{0}\left(x_{e}\right)\right|^{2} \leq C \int_{T}\left|\nabla \phi_{0}\right|^{2} d x
$$

Thus, we have

$$
\begin{aligned}
\left|\phi_{I}\left(x_{i}\right)-\phi_{I}\left(x_{k}\right)\right|^{2} & =\left|\bar{\phi}_{0}\right|_{T}-\left.\left.\bar{\phi}_{0}\right|_{T_{*}}\right|^{2} \\
& =\left|\bar{\phi}_{0}\right|_{T}-\left.\phi_{0}\right|_{T}\left(x_{e}\right)+\llbracket \phi_{0} \rrbracket\left(x_{e}\right)+\left.\phi_{0}\right|_{T_{*}}\left(x_{e}\right)-\left.\left.\bar{\phi}_{0}\right|_{T_{*}}\right|^{2} \\
& \leq C \sum_{e \subset \partial T} h_{e}^{-1}\left\|\llbracket \phi_{0} \rrbracket\right\|_{e}^{2}+C \int_{T \cup T_{*}}\left|\nabla \phi_{0}\right|^{2} d x
\end{aligned}
$$

Substituting the above estimate into (15) yields

$$
\left\|\nabla \phi_{I}\right\|^{2} \leq C \sum_{T}\left(\left\|\nabla \phi_{0}\right\|_{T}^{2}+h^{-1}\left\|\llbracket \phi_{0} \rrbracket\right\|_{\partial T}^{2}\right) .
$$

By combining (13) with (14) and (16) we obtain

$$
\|\phi\|^{2} \leq C \sum_{T}\left(\left\|\nabla \phi_{0}\right\|_{T}^{2}+h^{-1}\left\|\phi_{0}-\phi_{b}\right\|_{\partial T}^{2}\right)
$$

where (12) has been applied to estimate the jump of $\phi_{0}$ on each edge.

Next, we want to bound the two terms on the right hand side of (17) by $\left\|\nabla_{d} \phi\right\|$. Let us recall that the weak gradient $\nabla_{d} \phi$ is defined by

$$
\int_{T} \nabla_{d} \phi \cdot \mathbf{q} d x=-\int_{T} \phi_{0} \nabla \cdot \mathbf{q} d x+\int_{\partial T} \phi_{b} \mathbf{q} \cdot \mathbf{n} d s, \quad \forall \mathbf{q} \in V(T, j+1),
$$

and by using integration by parts, we have

$$
\int_{T} \nabla_{d} \phi \cdot \mathbf{q} d x=\int_{T} \nabla \phi_{0} \cdot \mathbf{q} d x-\int_{\partial T}\left(\phi_{0}-\phi_{b}\right) \mathbf{q} \cdot \mathbf{n} d s, \quad \forall \mathbf{q} \in V(T, j+1) .
$$

In order to bound $\left\|\nabla \phi_{0}\right\|$ and $\left\|\llbracket \phi_{0} \rrbracket\right\|_{e}$ by $\left\|\nabla_{d} \phi\right\|$, we let $\mathbf{q}$ in (18) satisfy the following

$$
\begin{aligned}
\int_{T} \mathbf{q} \cdot \mathbf{r} d x & =\int_{T} \nabla \phi_{0} \cdot \mathbf{r} d x \quad \forall \mathbf{r} \in\left[P_{j}(T)\right]^{2} \\
\int_{\partial T} \mathbf{q} \cdot \mathbf{n} \mu d s & =-h^{-1} \int_{\partial T}\left(\phi_{0}-\phi_{b}\right) \mu d s \quad \forall \mu \in P_{j+1}[\partial T],
\end{aligned}
$$

where by Lemma 5.1 in [12], q and $\phi$ have the norm equivalence of

$$
\|\mathbf{q}\|_{L^{2}(T)} \approx\left\|\nabla \phi_{0}\right\|_{L^{2}(T)}+h^{-\frac{1}{2}}\left\|\phi_{0}-\phi_{b}\right\|_{L^{2}(\partial T)} .
$$

Then from (18), we have

$$
\begin{aligned}
\int_{T} \nabla_{d} \phi \cdot \mathbf{q} d x & =\int_{T} \nabla \phi_{0} \cdot \mathbf{q} d x-\int_{\partial T}\left(\phi_{0}-\phi_{b}\right) \mathbf{q} \cdot \mathbf{n} d s \\
& =\int_{T} \nabla \phi_{0} \cdot \nabla \phi_{0} d x+h^{-1} \int_{\partial T}\left(\phi_{0}-\phi_{b}\right)^{2} d s \\
& =\left\|\nabla \phi_{0}\right\|_{T}^{2}+h^{-1}\left\|\phi_{0}-\phi_{b}\right\|_{\partial T}^{2} .
\end{aligned}
$$

Using (18) and (19), we have

$$
\begin{aligned}
\left\|\nabla_{d} \phi\right\|_{T} \geq \frac{1}{\|\mathbf{q}\|}\left(\nabla_{d} \phi, \mathbf{q}\right)_{T} & =\frac{1}{\|\mathbf{q}\|}\left(\left\|\nabla \phi_{0}\right\|_{T}^{2}+h^{-1}\left\|\phi_{0}-\phi_{b}\right\|_{\partial T}^{2}\right) \\
& \geq \underset{8}{C}\left(\left\|\nabla \phi_{0}\right\|_{T}^{2}+h^{-1}\left\|\phi_{0}-\phi_{b}\right\|_{\partial T}^{2}\right),
\end{aligned}
$$


then together with (17), we have the assertion.

With the results established in Lemma 4.2, we are ready to derive an error estimate for the discrete time weak Galerkin approximation $u_{h}$ as the following theorem.

Theorem 4.2. Let $u \in H^{1+r}(\Omega)$ and $U^{n}$ be the solutions of (1) and (7), respectively. Denote by $e^{n}:=U^{n}-Q_{h} u\left(t_{n}\right)$ the difference between the backward Euler weak Galerkin approximation and the $L^{2}$ projection of the exact solution $u$. Then there exists a constant $C$ such that

$$
\left\|e^{n}\right\|^{2}+\sum_{j=1}^{n} \alpha k\left\|\nabla_{d} e^{j}\right\|^{2} \leq\left\|e^{0}\right\|^{2}+C\left(h^{2 r}\|u\|_{1+r}^{2}+k^{2} \int_{0}^{t_{n}}\left\|u_{t t}\right\|^{2} d s\right), \quad \text { for } n \geq 0
$$

and

$$
\left\|\nabla_{d} e^{n}\right\|^{2} \leq C\left(\left\|e^{0}\right\|^{2}+\left\|\nabla_{d} e^{0}\right\|^{2}+h^{2 r}\left(\|u\|_{1+r}^{2}+\left\|u_{t}\right\|_{1+r}^{2}\right)+k^{2} \int_{0}^{t_{n}}\left\|u_{t t}\right\|^{2} d s\right),
$$

where $\|u\|_{1+r}=\max _{j=1, \cdots, n}\left\{\left\|u\left(t_{j}\right)\right\|_{1+r}\right\}$ and $\left\|u_{t}\right\|_{1+r}=\max _{j=1, \cdots, n}\left\{\left\|u_{t}\left(t_{j}\right)\right\|_{1+r}\right\}$.

Proof. A calculation corresponding to the error equation (10) yields

$$
\begin{aligned}
& \left.\left(\bar{\partial}\left(U^{n}-Q_{h} u\left(t_{n}\right)\right), v_{0}\right)+a\left(U^{n}-Q_{h} u\left(t_{n}\right)\right), v\right) \\
= & \left(u_{t}\left(t_{n}\right)-\bar{\partial} u\left(t_{n}\right), v_{0}\right)+\left(\Pi_{h}\left(a \nabla u\left(t_{n}\right)\right)-a \mathbb{Q}_{h}\left(\nabla u\left(t_{n}\right)\right), \nabla_{d} v\right),
\end{aligned}
$$

i.e.,

$$
\left(\bar{\partial} e^{n}, v_{0}\right)+a\left(e^{n}, v\right)=\left(u_{t}\left(t_{n}\right)-\bar{\partial} u\left(t_{n}\right), v_{0}\right)+\left(\Pi_{h}\left(a \nabla u\left(t_{n}\right)\right)-a \mathbb{Q}_{h}\left(\nabla u\left(t_{n}\right)\right), \nabla_{d} v\right) .
$$

Let $w_{1}^{n}=u_{t}\left(t_{n}\right)-\bar{\partial} u\left(t_{n}\right)$, and $w_{2}^{n}=\Pi_{h}\left(a \nabla u\left(t_{n}\right)\right)-a \mathbb{Q}_{h}\left(\nabla u\left(t_{n}\right)\right)$, and choosing $v=e^{n}$ in (20), we have

$$
\left(\bar{\partial} e^{n}, e^{n}\right)+a\left(e^{n}, e^{n}\right)=\left(w_{1}^{n}, e^{n}\right)+\left(w_{2}^{n}, \nabla_{d} e^{n}\right) .
$$

By coercivity of the bilinear form and Cauchy-Schwarz inequality, we obtain

$$
\left\|e^{n}\right\|^{2}-\left(e^{n-1}, e^{n}\right)+\alpha k\left\|\nabla_{d} e^{n}\right\|^{2} \leq k\left\|w_{1}^{n}\right\|\left\|e^{n}\right\|+k\left\|w_{2}^{n}\right\|\left\|\nabla_{d} e^{n}\right\|,
$$

i.e.,

$$
\left\|e^{n}\right\|^{2}+\alpha k\left\|\nabla_{d} e^{n}\right\|^{2} \leq \frac{1}{2}\left\|e^{n-1}\right\|^{2}+\frac{1}{2}\left\|e^{n}\right\|^{2}+k\left\|w_{1}^{n}\right\|\left\|e^{n}\right\|+k\left\|w_{2}^{n}\right\|\left\|\nabla_{d} e^{n}\right\| .
$$

By the Poincaré inequality of Lemma 4.2, we have

$$
\frac{1}{2}\left\|e^{n}\right\|^{2}+\alpha k\left\|\nabla_{d} e^{n}\right\|^{2} \leq \frac{1}{2}\left\|e^{n-1}\right\|^{2}+k\left(C\left\|w_{1}^{n}\right\|+\left\|w_{2}^{n}\right\|\right)\left(\left\|\nabla_{d} e^{n}\right\|\right) .
$$

Then by triangle inequality, it follows

so that

$$
\frac{1}{2}\left\|e^{n}\right\|^{2}+\frac{\alpha k}{2}\left\|\nabla_{d} e^{n}\right\|^{2} \leq \frac{1}{2}\left\|e^{n-1}\right\|^{2}+\frac{k}{2 \alpha}\left(C\left\|w_{1}^{n}\right\|+\left\|w_{2}^{n}\right\|\right)^{2} .
$$

$$
\left\|e^{n}\right\|^{2}+\alpha k\left\|\nabla_{d} e^{n}\right\|^{2} \leq\left\|e^{n-1}\right\|^{2}+\frac{C k}{\alpha}\left\|w_{1}^{n}\right\|^{2}+\frac{k}{\alpha}\left\|w_{2}^{n}\right\|^{2} .
$$

and, by repeated application,

$$
\left\|e^{n}\right\|^{2}+\sum_{j=1}^{n} \alpha k\left\|\nabla_{d} e^{j}\right\|^{2} \leq\left\|e^{0}\right\|^{2}+\frac{C k}{\alpha} \sum_{j=1}^{n}\left\|w_{1}^{j}\right\|^{2}+\frac{k}{\alpha} \sum_{j=1}^{n}\left\|w_{2}^{j}\right\|^{2} .
$$

We write

$$
k w_{1}^{j}=k u_{t}\left(t_{j}\right)-\left(u\left(t_{j}\right)-u\left(t_{j-1}\right)\right)=\int_{t_{j-1}}^{t_{j}}\left(s-t_{j-1}\right) u_{t t}(s) d s
$$


i.e.,

$$
w_{1}^{j}=u_{t}\left(t_{j}\right)-\frac{u\left(t_{j}\right)-u\left(t_{j-1}\right)}{k}=\frac{1}{k} \int_{t_{j-1}}^{t_{j}}\left(s-t_{j-1}\right) u_{t t}(s) d s,
$$

so that

$$
\begin{aligned}
\left\|w_{1}^{j}\right\|^{2} & =\int_{\Omega}\left\{\frac{1}{k} \int_{t_{j-1}}^{t_{j}}\left(s-t_{j-1}\right) u_{t t}(s) d s\right\}^{2} d x \\
& \leq \frac{1}{k^{2}} \int_{\Omega} \int_{t_{j-1}}^{t_{j}}\left(s-t_{j-1}\right)^{2} d s \int_{t_{j-1}}^{t_{j}} u_{t t}^{2}(s) d s d x \\
& \leq C k \int_{t_{j-1}}^{t_{j}}\left\|u_{t t}\right\|^{2} d s .
\end{aligned}
$$

Substitute (23) into (21) and together with Lemma 4.1, we have the error estimate for $\left\|e^{n}\right\|$.

In order to show an estimate for $\nabla_{d} e^{n}$ we may choose instead $v=\bar{\partial} e^{n}$ in error equation (21) to obtain the following identity

$$
\left(\bar{\partial} e^{n}, \bar{\partial} e^{n}\right)+a\left(e^{n}, \bar{\partial} e^{n}\right)=\left(w_{1}^{n}, \bar{\partial} e^{n}\right)+\left(w_{2}^{n}, \nabla_{d} \bar{\partial} e^{n}\right) .
$$

The second term on the right hand side can be written as

$$
\left(w_{2}^{n}, \nabla_{d} \bar{\partial} e^{n}\right)=\bar{\partial}\left(w_{2}^{n}, \nabla_{d} e^{n}\right)-\left(\left(w_{2}^{n}\right)_{t}-\bar{\partial} w_{2}^{n}, \nabla_{d} e^{n-1}\right)+\left(\left(w_{2}^{n}\right)_{t}, \nabla_{d} e^{n-1}\right) .
$$

Then the error equation becomes

$$
\begin{aligned}
k\left\|\bar{\partial} e^{n}\right\|^{2}+\left(a \nabla_{d} e^{n}, \nabla_{d} e^{n}\right) & =\left(a \nabla_{d} e^{n}, \nabla_{d} e^{n-1}\right)+k\left(w_{1}, \bar{\partial} e^{n}\right) \\
& +k \bar{\partial}\left(w_{2}^{n}, \nabla_{d} e^{n}\right)-k\left(\left(w_{2}^{n}\right)_{t}-\bar{\partial} w_{2}^{n}, \nabla_{d} e^{n-1}\right)+k\left(\left(w_{2}^{n}\right)_{t}, \nabla_{d} e^{n-1}\right) .
\end{aligned}
$$

By triangle inequality, we have

$$
\begin{aligned}
k\left\|\bar{\partial} e^{n}\right\|^{2}+\left(a \nabla_{d} e^{n}, \nabla_{d} e^{n}\right) & \leq \frac{1}{2}\left(a \nabla_{d} e^{n}, \nabla_{d} e^{n}\right)+\frac{1}{2}\left(a \nabla_{d} e^{n-1}, \nabla_{d} e^{n-1}\right) \\
& +\frac{k}{4}\left\|w_{1}^{n}\right\|^{2}+k\left\|\bar{\partial} e^{n}\right\|^{2}+k \bar{\partial}\left(w_{2}^{n}, \nabla_{d} e^{n}\right) \\
& +\frac{k}{2}\left\|\left(w_{2}^{n}\right)_{t}-\bar{\partial} w_{2}^{n}\right\|^{2}+\frac{k}{2}\left\|\nabla_{d} e^{n-1}\right\|^{2} \\
& +\frac{k}{2}\left\|\left(w_{2}^{n}\right)_{t}\right\|^{2}+\frac{k}{2}\left\|\nabla_{d} e^{n-1}\right\|^{2},
\end{aligned}
$$

and, after cancelation and by repeated application,

$$
\begin{aligned}
\frac{1}{2}\left(a \nabla_{d} e^{n}, \nabla_{d} e^{n}\right) & \leq \frac{1}{2}\left(a \nabla_{d} e^{0}, \nabla_{d} e^{0}\right) \\
& +\frac{k}{4} \sum_{j=1}^{n}\left\|w_{1}^{j}\right\|^{2}+\left(w_{2}^{n}, \nabla_{d} e^{n}\right)-\left(w_{2}^{0}, \nabla_{d} e^{0}\right) \\
& +\frac{k}{2} \sum_{j=1}^{n}\left\|\left(w_{2}^{n}\right)_{t}-\bar{\partial} w_{2}^{j}\right\|^{2}+\frac{k}{2} \sum_{j=1}^{n}\left\|\left(w_{2}^{n}\right)_{t}\right\|^{2}+k \sum_{j=1}^{n}\left\|\nabla_{d} e^{j-1}\right\|^{2}
\end{aligned}
$$


which is

$$
\begin{aligned}
\frac{\alpha}{2}\left\|\nabla_{d} e^{n}\right\|^{2} & \leq \frac{\beta}{2}\left\|\nabla_{d} e^{0}\right\|^{2} \\
& +\frac{k}{4} \sum_{j=1}^{n}\left\|w_{1}^{j}\right\|^{2}+\frac{1}{\alpha}\left\|w_{2}^{n}\right\|^{2}+\frac{\alpha}{4}\left\|\nabla_{d} e^{n}\right\|^{2}+\frac{1}{2 \beta}\left\|w_{2}^{0}\right\|^{2}+\frac{\beta}{2}\left\|\nabla_{d} e^{0}\right\|^{2} \\
& +\frac{k}{2} \sum_{j=1}^{n}\left\|\left(w_{2}^{n}\right)_{t}-\bar{\partial} w_{2}^{j}\right\|^{2}+\frac{k}{2} \sum_{j=1}^{n}\left\|\left(w_{2}^{n}\right)_{t}\right\|^{2}+k \sum_{j=1}^{n}\left\|\nabla_{d} e^{j-1}\right\|^{2}
\end{aligned}
$$

followed by triangle inequality and boundness and coercivity of the bilinear form. By the similar process as in (23) and Lemma 4.1, we have

$$
\left\|\left(w_{2}^{n}\right)_{t}-\bar{\partial} w_{2}^{j}\right\|^{2} \leq C k \int_{t_{j-1}}^{t_{j}}\left\|\left(w_{2}\right)_{t t}\right\|^{2} d s \leq C k h^{2 r} \int_{t_{j-1}}^{t_{j}}\left\|u_{t t}\right\|_{1+r}^{2} d s .
$$

Then by substituting (23), (21) and the above inequality into (24), we have the error estimate for $\left\|\nabla_{d} e^{n}\right\|$ as the following

$$
\left\|\nabla_{d} e^{n}\right\|^{2} \leq C\left(\left\|e^{0}\right\|^{2}+\left\|\nabla_{d} e^{0}\right\|^{2}+h^{2 r}\left(\|u\|_{1+r}^{2}+\left\|u_{t}\right\|_{1+r}^{2}\right)+k^{2} \int_{0}^{t_{n}}\left\|u_{t t}\right\|^{2} d s\right),
$$

which completes the proof.

4.3. Optimal Order of Error Estimation in $L^{2}$. To get an optimal order of error estimate in $L^{2}$, the idea, similar to Wheeler's projection as in [13] and [1], is used where an elliptic projection $E_{h}$ onto the discrete weak space $S_{h}(j, l)$ is defined as the following: Find $E_{h} v \in S_{h}(j, l)$ such that $E_{h} v$ is the $L^{2}$ projection of the trace of $v$ on the boundary $\partial \Omega$ and

$$
\left(a \nabla_{d} E_{h} v, \nabla_{d} \chi\right)=(-\nabla \cdot(a \nabla v), \chi), \quad \forall \chi \in S_{h}^{0}(j, l) .
$$

In view of the weak formulation of the elliptic problem,

$$
\begin{array}{rlr}
-\nabla \cdot(a \nabla v) & =F \quad \text { in } \Omega, \\
v & =g \quad \text { on } \partial \Omega,
\end{array}
$$

this definition may be expressed by saying that $E_{h} v$ is the weak Galerkin finite element approximation of the solution of the corresponding elliptic problem with exact solution $v$. By the error estimate results in [7, we have the following error estimate for $E_{h} v$.

Lemma 4.3. Assume that problem (26) has the $H^{1+s}$ regularity $(s \in(0,1])$. Let $v \in H^{1+r}$ be the exact solution of (26), and $E_{h} v$ be a weak Galerkin approximation of $v$ defined in (25). Let $Q_{h} v=\left\{Q_{0} v, Q_{b} v\right\}$ be the $L^{2}$ projection of $v$ in the corresponding finite element space. Then there exists a constant $C$ such that

$$
\left\|Q_{0} v-E_{h} v\right\| \leq C\left(h^{s+1}\left\|F-Q_{0} F\right\|+h^{r+s}\|v\|_{r+1}\right),
$$

and

$$
\left\|\nabla_{d}\left(Q_{h} v-E_{h} v\right)\right\| \leq C h^{r}\|v\|_{r+1}
$$

Throughout this section the error in the parabolic problem (11) is written as a sum of two terms,

$$
u_{h}(t)-Q_{h} u(t)=\theta(t)+\rho(t), \quad \text { where } \theta=u_{h}-E_{h} u, \quad \rho=E_{h} u-Q_{h} u,
$$

which will be bounded separately. Notice that the second term is the error in an elliptic problem and then can be handled by applying the results in Lemma 4.3. Then our main goal here is to bound the first term $\theta$.

Following the above strategy, the error estimate for continuous time weak Galerkin finite element method in $L^{2}$ and $H^{1}$ are provided in the next two theorems. 
Theorem 4.3. Under the assumption of Theorem 4.1 and the assumption that the corresponding elliptic problem has the $H^{1+s}$ regularity $(s \in(0,1])$, there exists a constant $C$ such that

$$
\begin{aligned}
\left\|u_{h}(t)-Q_{h} u(t)\right\| & \leq\left\|u_{h}(0)-Q_{h} u(0)\right\|+C h^{r+s}\left(\|\psi\|_{r+1}+\int_{0}^{t}\left\|u_{t}\right\|_{r+1} d s\right) \\
& +C h^{s+1}\left(\left\|f(0)-Q_{0} f(0)\right\|+\left\|u_{t}(0)-Q_{0} u_{t}(0)\right\|\right) \\
& +C h^{s+1}\left\{\int_{0}^{t}\left(\left\|f_{t}-Q_{0} f_{t}\right\|+\left\|u_{t t}-Q_{0} u_{t t}\right\|\right) d s\right\} .
\end{aligned}
$$

Proof. We write the error according to (29) and obtain the error bound for $\rho$ easily by Lemma 4.3 as the following

$$
\|\rho\| \leq C\left(h^{s+1}\left(\left\|f-Q_{0} f\right\|+\left\|u_{t}-Q_{0} u_{t}\right\|\right)+h^{r+s}\left(\|\psi\|_{r+1}+\int_{0}^{t}\left\|u_{t}\right\|_{r+1} d s\right)\right) .
$$

In order to estimate $\theta$, we note that by our definitions

$$
\begin{aligned}
\left(\theta_{t}, \chi\right)+a(\theta, \chi) & =\left(u_{h, t}, \chi\right)+a\left(u_{h}, \chi\right)-\left(E_{h} u_{t}, \chi\right)-a\left(E_{h} u, \chi\right) \\
& =(f, \chi)-\left(E_{h} u_{t}, \chi\right)-a\left(E_{h} u, \chi\right) \\
& =(f, \chi)+(\nabla \cdot(a \nabla u), \chi)-\left(E_{h} u_{t}, \chi\right) \\
& =\left(u_{t}, \chi\right)-\left(E_{h} u_{t}, \chi\right) \\
& =\left(Q_{h} u_{t}, \chi\right)-\left(E_{h} u_{t}, \chi\right) \\
& =-\left(\rho_{t}, \chi\right)
\end{aligned}
$$

which is

$$
\left(\theta_{t}, \chi\right)+a(\theta, \chi)=-\left(\rho_{t}, \chi\right), \quad \forall \chi \in S_{h}^{0}(j, l), t>0,
$$

where we have used the fact that the operator $E_{h}$ commutes with time differentiation. Since $\theta \in S_{h}^{0}(j, l)$, we may choose $\chi=\theta$ in (31) and obtain

$$
\left(\theta_{t}, \theta\right)+a(\theta, \theta)=-\left(\rho_{t}, \theta\right), \quad t>0 .
$$

Since

$$
a(\theta, \theta) \geq \alpha\left\|\nabla_{d} \theta\right\|^{2}>0
$$

we have

and hence

$$
\frac{1}{2} \frac{d}{d t}\|\theta\|^{2}=\|\theta\| \frac{d}{d t}\|\theta\| \leq\left\|\rho_{t}\right\|\|\theta\|
$$

Using Lemma 4.3, we find

$$
\|\theta(t)\| \leq\|\theta(0)\|+\int_{0}^{t}\left\|\rho_{t}\right\| d s .
$$

$$
\begin{aligned}
\|\theta(0)\| \leq & \left\|u_{h}(0)-E_{h} u(0)\right\| \\
\leq & \left\|u_{h}(0)-Q_{h} u(0)\right\|+\left\|E_{h} u(0)-Q_{h} u(0)\right\| \\
\leq & \left\|u_{h}(0)-Q_{h} u(0)\right\| \\
& +C\left[h^{s+1}\left(\left\|f(0)-Q_{0} f(0)\right\|+\left\|u_{t}(0)-Q_{0} u_{t}(0)\right\|\right)+h^{r+s}\|\psi\|_{r+1}\right],
\end{aligned}
$$

and since

$$
\left\|\rho_{t}\right\|=\left\|E_{h} u_{t}-Q_{h} u_{t}\right\| \leq C\left[h^{s+1}\left(\left\|f_{t}-Q_{0} f_{t}\right\|+\left\|u_{t t}-Q_{0} u_{t t}\right\|\right)+h^{r+s}\left\|u_{t}\right\|_{r+1}\right],
$$

the desired bound for $\|\theta(t)\|$ now follows. 
Theorem 4.4. Under the assumption of Theorem 4.3 and the assumption that the coefficient matrix $a$ in (1) is independent of time $t$, there exists a constant $C$ such that

$$
\begin{aligned}
\left\|\nabla_{d}\left(u_{h}(t)-Q_{h} u(t)\right)\right\|^{2} & \leq 4 \beta\left\|\nabla_{d}\left(u_{h}(0)-Q_{h} u(0)\right)\right\|^{2}+C h^{2 r}\left(\|\psi\|_{r+1}^{2}+\|u\|_{r+1}^{2}\right) \\
& +C h^{2(s+1)} \int_{0}^{t}\left(\left\|f_{t}-Q_{0} f_{t}\right\|+\left\|u_{t t}-Q_{0} u_{t t}\right\|\right)^{2} d s+C h^{2(r+s)} \int_{0}^{t}\left\|u_{t}\right\|_{r+1}^{2} d s .
\end{aligned}
$$

Proof. As in the proof of Theorem 4.3, we write the error in the form (29). Here by Lemma 4.3,

$$
\left\|\nabla_{d} \rho(t)\right\| \leq C h^{r}\|u\|_{r+1} .
$$

In order to estimate $\nabla_{d} \theta$, we may choose $\chi=\theta_{t}$ in the equation (31) for $\theta$. We obtain

$$
\left(\theta_{t}, \theta_{t}\right)+a\left(\theta, \theta_{t}\right)=-\left(\rho_{t}, \theta_{t}\right) .
$$

Since the coefficient matrix $a$ in the bilinear form $a(\cdot, \cdot)$ is independent of time $t$, we have

$$
\left\|\theta_{t}\right\|^{2}+\frac{1}{2} \frac{d}{d t}\left(a \nabla_{d} \theta, \nabla_{d} \theta\right)=-\left(\rho_{t}, \theta_{t}\right) \leq \frac{1}{2}\left\|\rho_{t}\right\|^{2}+\frac{1}{2}\left\|\theta_{t}\right\|^{2},
$$

so that

$$
\frac{d}{d t}\left(a \nabla_{d} \theta, \nabla_{d} \theta\right) \leq\left\|\rho_{t}\right\|^{2}
$$

Then by integrating with respect to time $t$ and using the coercivity and boundedness of the bilinear form, we obtain

$$
\begin{aligned}
\alpha\left\|\nabla_{d} \theta\right\|^{2} & \leq\left(a \nabla_{d} \theta, \nabla_{d} \theta\right) \leq\left(a \nabla_{d} \theta(0), \nabla_{d} \theta(0)\right)+\int_{0}^{t}\left\|\rho_{t}\right\|^{2} d s \leq \beta\left\|\nabla_{d} \theta(0)\right\|^{2}+\int_{0}^{t}\left\|\rho_{t}\right\|^{2} d s \\
& \leq \beta\left(\left\|\nabla_{d}\left(u_{h}(0)-Q_{h} u(0)\right)\right\|+\left\|\nabla_{d}\left(E_{h} u(0)-Q_{h} u(0)\right)\right\|\right)^{2}+\int_{0}^{t}\left\|\rho_{t}\right\|^{2} d s .
\end{aligned}
$$

Hence, in view of Lemma 4.3 and (33), we have

$$
\begin{aligned}
\left\|\nabla_{d} \theta(t)\right\|^{2} \leq & 2 \beta\left\|\nabla_{d}\left(u_{h}(0)-Q_{h} u(0)\right)\right\|^{2}+C h^{2 r}\|\psi\|_{r+1}^{2} \\
& +C h^{2(s+1)} \int_{0}^{t}\left(\left\|f_{t}-Q_{0} f_{t}\right\|+\left\|u_{t t}-Q_{0} u_{t t}\right\|\right)^{2} d s+C h^{2(r+s)} \int_{0}^{t}\left\|u_{t}\right\|_{r+1}^{2} d s,
\end{aligned}
$$

which completes the proof.

Next, we derive an error estimate for the backward Euler weak Galerkin method.

Theorem 4.5. Let $u \in H^{1+r}(\Omega)$ and $U^{n}$ be the solutions of (1) and (7), respectively. And let $Q_{h} u$ be the $L^{2}$ projection of the exact solution $u$. Then there exists a constant $C$ such that

$$
\begin{aligned}
\left\|U^{n}-Q_{h} u\left(t_{n}\right)\right\| & \leq\left\|U^{0}-Q_{h} u(0)\right\|+C h^{r+s}\left(\|\psi\|_{r+1}+\int_{0}^{t_{n}}\left\|u_{t}\right\|_{r+1} d s\right) \\
& +C h^{s+1}\left(\left\|f(0)-Q_{0} f(0)\right\|+\left\|u_{t}(0)-Q_{0} u_{t}(0)\right\|\right) \\
& +C h^{s+1}\left(\left\|f\left(t_{n}\right)-Q_{0} f\left(t_{n}\right)\right\|+\left\|u_{t}\left(t_{n}\right)-Q_{0} u_{t}\left(t_{n}\right)\right\|\right) \\
& +C h^{s+1}\left\{\int_{0}^{t_{n}}\left(\left\|f_{t}-Q_{0} f_{t}\right\|+\left\|u_{t t}-Q_{0} u_{t t}\right\|\right) d s\right\} \\
& +C k \int_{0}^{t_{n}}\left\|u_{t t}\right\| d s .
\end{aligned}
$$


Proof. In analogy with (29), we write

$$
U^{n}-Q_{h} u\left(t_{n}\right)=\left(U^{n}-E_{h} u\left(t_{n}\right)\right)+\left(E_{h} u\left(t_{n}\right)-Q_{h} u\left(t_{n}\right)\right)=\theta^{n}+\rho^{n},
$$

where $\rho^{n}=\rho\left(t_{n}\right)$ is bounded as shown in (30). In order to bound $\theta^{n}$, we use

$$
\begin{aligned}
\left(\bar{\partial} \theta^{n}, \chi\right)+a\left(\theta^{n}, \chi\right) & =\left(\bar{\partial} U^{n}, \chi\right)+a\left(U^{n}, \chi\right)-\left(\bar{\partial} E_{h} u\left(t_{n}\right), \chi\right)-a\left(E_{h} u\left(t_{n}\right), \chi\right) \\
& =\left(f\left(t_{n}\right), \chi\right)-\left(\bar{\partial} E_{h} u\left(t_{n}\right), \chi\right)-a\left(E_{h} u\left(t_{n}\right), \chi\right) \\
& =\left(f\left(t_{n}\right), \chi\right)+\left(\nabla \cdot\left(a \nabla u\left(t_{n}\right)\right), \chi\right)-\left(\bar{\partial} E_{h} u\left(t_{n}\right), \chi\right) \\
& =\left(u_{t}\left(t_{n}\right), \chi\right)-\left(\bar{\partial} E_{h} u\left(t_{n}\right), \chi\right) \\
& =\left(u_{t}\left(t_{n}\right)-\bar{\partial} u\left(t_{n}\right), \chi\right)+\left(\bar{\partial} u\left(t_{n}\right)-\bar{\partial} E_{h} u\left(t_{n}\right), \chi\right),
\end{aligned}
$$

i.e.,

$$
\left(\bar{\partial} \theta^{n}, \chi\right)+a\left(\theta^{n}, \chi\right)=\left(w^{n}, \chi\right)
$$

where

$$
w^{n}=\left(u_{t}\left(t_{n}\right)-\bar{\partial} u\left(t_{n}\right)\right)+\left(\bar{\partial} u\left(t_{n}\right)-\bar{\partial} E_{h} u\left(t_{n}\right)\right)=w_{1}^{n}+w_{3}^{n} .
$$

By choosing $\chi=\theta^{n}$ in (35) and the coercivity of the bilinear form, we have

$$
\left(\bar{\partial} \theta^{n}, \theta^{n}\right) \leq\left\|w^{n}\right\|\left\|\theta^{n}\right\|
$$

or

$$
\left\|\theta^{n}\right\|^{2}-\left(\theta^{n-1}, \theta^{n}\right) \leq k\left\|w^{n}\right\|\left\|\theta^{n}\right\|
$$

so that

$$
\left\|\theta^{n}\right\| \leq\left\|\theta^{n-1}\right\|+k\left\|w^{n}\right\|,
$$

and, by repeated application, it follows

$$
\left\|\theta^{n}\right\| \leq\left\|\theta^{0}\right\|+k \sum_{j=1}^{n}\left\|w^{j}\right\| \leq\left\|\theta^{0}\right\|+k \sum_{j=1}^{n}\left\|w_{1}^{j}\right\|+k \sum_{j=1}^{n}\left\|w_{3}^{j}\right\| .
$$

As in (32),$\theta^{0}=\theta(0)$ is bounded as desired. By using the representation in (22), we obtain

$$
k \sum_{j=1}^{n}\left\|w_{1}^{j}\right\| \leq \sum_{j=1}^{n}\left\|\int_{t_{j-1}}^{t_{j}}\left(s-t_{j-1}\right) u_{t t}(s) d s\right\| \leq k \int_{0}^{t_{n}}\left\|u_{t t}\right\| d s .
$$

We write

$$
w_{3}^{j}=\bar{\partial} u\left(t_{n}\right)-E_{h} \bar{\partial} u\left(t_{n}\right)=\left(E_{h}-I\right) k^{-1} \int_{t_{j-1}}^{t_{j}} u_{t} d s=k^{-1} \int_{t_{j-1}}^{t_{j}}\left(E_{h}-I\right) u_{t} d s,
$$

and, by (33) we have

$$
\begin{aligned}
k \sum_{j=1}^{n}\left\|w_{3}^{j}\right\| & \leq \sum_{j=1}^{n} \int_{t_{j-1}}^{t_{j}} C\left[h^{s+1}\left(\left\|f_{t}-Q_{0} f_{t}\right\|+\left\|u_{t t}-Q_{0} u_{t t}\right\|\right)+h^{r+s}\left\|u_{t}\right\|_{r+1}\right] d s \\
& =C\left[h^{s+1} \int_{0}^{t_{n}}\left(\left\|f_{t}-Q_{0} f_{t}\right\|+\left\|u_{t t}-Q_{0} u_{t t}\right\|\right) d s+h^{r+s} \int_{0}^{t_{n}}\left\|u_{t}\right\|_{r+1} d s\right],
\end{aligned}
$$

Thus, together with the estimate of $\rho$ in (30), we have the assertion. 
Theorem 4.6. Under the assumption of Theorem 4.5, and the assumption that the coefficient matrix $a$ is independent of time $t$, there exists a constant $C$ such that

$$
\begin{aligned}
\left\|\nabla_{d}\left(U^{n}-Q_{h} u\left(t_{n}\right)\right)\right\|^{2} & \leq 2\left\|\nabla_{d}\left(U^{0}-Q_{h} u(0)\right)\right\|^{2}+C h^{2 r}\left(\|\psi\|_{r+1}^{2}+\|u\|_{r+1}^{2}\right) \\
& +C h^{2(s+1)} \int_{0}^{t_{n}}\left(\left\|f_{t}-Q_{0} f_{t}\right\|+\left\|u_{t t}-Q_{0} u_{t t}\right\|\right)^{2} d s+C h^{2(r+s)} \int_{0}^{t_{n}}\left\|u_{t}\right\|_{r+1}^{2} d s \\
& +C k^{2} \int_{0}^{t_{n}}\left\|u_{t t}\right\|^{2} d s .
\end{aligned}
$$

Proof. It is sufficient to estimate $\nabla_{d} \theta^{n}$. To this end, we choose $\chi=\bar{\partial} \theta^{n}$ in (35), and it is easily seen that

$$
\left(\bar{\partial} \theta^{n}, \bar{\partial} \theta^{n}\right)+a\left(\theta^{n}, \bar{\partial} \theta^{n}\right)=\left\|\bar{\partial} \theta^{n}\right\|^{2}+\frac{1}{2} \bar{\partial} a\left(\theta^{n}, \theta^{n}\right)+\frac{k}{2} a\left(\bar{\partial} \theta^{n}, \bar{\partial} \theta^{n}\right)=\left(w^{n}, \bar{\partial} \theta^{n}\right),
$$

so that

$$
\bar{\partial} a\left(\theta^{n}, \theta^{n}\right) \leq\left\|w^{n}\right\|^{2} .
$$

By repeating the application, we have

$$
\begin{aligned}
a\left(\theta^{n}, \theta^{n}\right) & \leq a\left(\theta^{0}, \theta^{0}\right)+k \sum_{j=0}^{n}\left\|w^{j}\right\|^{2} \\
& \leq a\left(\theta^{0}, \theta^{0}\right)+2 k \sum_{j=0}^{n}\left\|w_{1}^{j}\right\|^{2}+2 k \sum_{j=0}^{n}\left\|w_{3}^{j}\right\|^{2} .
\end{aligned}
$$

As in (36), we obtain

$$
k\left\|w_{3}^{j}\right\|^{2}=k \int_{\Omega}\left(k^{-1} \int_{t_{j-1}}^{t_{j}} \rho_{t} d s\right)^{2} d x \leq \int_{\Omega}\left(\int_{t_{j-1}}^{t_{j}} \rho_{t}^{2} d s\right) d x \leq \int_{t_{j-1}}^{t_{j}}\left\|\rho_{t}\right\|^{2} d s .
$$

Together with (23), (33) and (34), we have the assertion.

\section{Numerical Experiments}

In section 2 , we mentioned that the discrete weak space $S_{h}(j, l)$ and $\sum_{h}$ in the weak Galerkin method need to satisfy two conditions. In [7], the authors proposed several possible combinations of $S_{h}(j, l)$ and $\sum_{h}$. Through out this section we use a uniform triangular mesh $\mathcal{T}_{h}$, the discrete weak space $S_{h}(0,0)$, i.e., space consisting of piecewise constants on the triangles and edges respectively, and $\sum_{h}$ with $V(T, 1)$ to be the lowest order Raviart-Thomas element $R T_{0}(T)$ in the weak Galerkin method, which were used in [14 for the numerical studies of the weak Galerkin method for secondorder elliptic problems. We also adopt the various norms used in 14 to present the numerical results of the error $e_{h}$ between the $L^{2}$ projection $Q_{h} u$ of the exact solution and the numerical solution $u_{h}$.

Example 1. As the first example, we consider the following heat equation in $\Omega=(0,1) \times(0,1)$,

$$
\begin{aligned}
& u_{t}-\nabla \cdot(a \nabla u)=f \quad \text { in } \Omega, \quad \text { for } t>0, \\
& u=g \text { on } \partial \Omega, \quad \text { for } t>0, \\
& u(\cdot, 0)=\psi \quad \text { in } \Omega \text {, }
\end{aligned}
$$

where $a=\left[\begin{array}{ll}1 & 0 \\ 0 & 1\end{array}\right]$ and $f, g$ and $\psi$ are determined by setting the exact solution $u=\sin \left(2 \pi\left(t^{2}+\right.\right.$ 1) $+\pi / 2) \sin (2 \pi x+\pi / 2) \sin (2 \pi y+\pi / 2)$. 
For this inhomogeneous Dirichlet boundary condition, with a uniform triangular mesh $\mathcal{T}_{h}$, we chose the approximation space

$$
S_{h}=\left\{\begin{array}{r}
v_{0} \in P_{0}(T), \quad \text { for all } T \in \mathcal{T}_{h}, \\
v=\left\{v_{0}, v_{b}\right\}: \quad v_{b} \in P_{0}(e) \text { for all } T \in \mathcal{T}_{h} \text { and } e \subset \partial T \notin \partial \Omega, \\
v_{b}=g_{h} \quad \text { for all } T \in \mathcal{T}_{h} \text { and } \partial T \in \partial \Omega
\end{array}\right\}
$$

where $g_{h}$ is the $L^{2}$ projection of $g$ in the piecewise constant finite element space on the boundary $\partial \Omega$. In the test, $k=h$ and $k=h^{2}$ are used to check the order of convergency with respect to time step size $k$ and mesh size $h$ respectively, since the convergence rate of the error is dominated by that of the time step size $k$ when $k=h$, and the convergence rate of the error is dominated by that of the mesh size $h$ when $k=h^{2}$. The results are shown in Table 1 and Table 2. Since the exact solution is smooth, we observe the optimal convergence rates in both $L^{2}$ and weak Galerkin $H^{1}$ norms for the Dirichlet boundary data type initial boundary value problem, which is consistent with the theoretical results shown in section 4 and 5 .

TABLE 1. Convergence rate for heat equation with inhomogeneous Dirichlet boundary condition with $k=h$

\begin{tabular}{|c|c|c|c|c|c|}
\hline$h$ & $\left\|e_{h}\right\|_{\{\infty, T\}}$ & $\left\|e_{h}\right\|_{\{\infty, \partial T\}}$ & $\left\|\nabla_{d} e_{h}\right\|$ & $\left\|e_{h}\right\|_{\left\{L^{2}, T\right\}}$ & $\left\|e_{h}\right\|_{\left\{L^{2}, \partial T\right\}}$ \\
\hline \hline $1 / 8$ & $1.38 \mathrm{e}-01$ & $1.44 \mathrm{e}-01$ & $2.41 \mathrm{e}-01$ & $4.90 \mathrm{e}-02$ & $8.78 \mathrm{e}-02$ \\
$1 / 16$ & $6.97 \mathrm{e}-02$ & $7.26 \mathrm{e}-02$ & $8.34 \mathrm{e}-02$ & $2.20 \mathrm{e}-02$ & $4.03 \mathrm{e}-02$ \\
$1 / 32$ & $3.47 \mathrm{e}-02$ & $3.56 \mathrm{e}-02$ & $2.97 \mathrm{e}-02$ & $1.05 \mathrm{e}-02$ & $1.94 \mathrm{e}-02$ \\
$1 / 64$ & $1.72 \mathrm{e}-02$ & $1.75 \mathrm{e}-02$ & $1.10 \mathrm{e}-02$ & $5.16 \mathrm{e}-03$ & $9.54 \mathrm{e}-03$ \\
$1 / 128$ & $8.59 \mathrm{e}-03$ & $8.65 \mathrm{e}-03$ & $4.27 \mathrm{e}-03$ & $2.56 \mathrm{e}-03$ & $4.74 \mathrm{e}-03$ \\
\hline \hline$O\left(h^{r}\right) r=$ & 1.0012 & 1.0138 & 1.4550 & 1.0643 & 1.0533 \\
\hline
\end{tabular}

TABLE 2. Convergence rate for heat equation with inhomogeneous Dirichlet boundary condition with $k=h^{2}$

\begin{tabular}{||c||c||c||c||c||c||}
\hline \hline$h$ & $\left\|e_{h}\right\|_{\{\infty, T\}}$ & $\left\|e_{h}\right\|_{\{\infty, \partial T\}}$ & $\left\|\nabla_{d} e_{h}\right\|$ & $\left\|e_{h}\right\|_{\left\{L^{2}, T\right\}}$ & $\left\|e_{h}\right\|_{\left\{L^{2}, \partial T\right\}}$ \\
\hline \hline $1 / 8$ & $7.11 \mathrm{e}-02$ & $8.30 \mathrm{e}-02$ & $7.81 \mathrm{e}-02$ & $3.28 \mathrm{e}-02$ & $5.39 \mathrm{e}-02$ \\
$1 / 16$ & $1.89 \mathrm{e}-02$ & $2.28 \mathrm{e}-02$ & $1.84 \mathrm{e}-02$ & $8.53 \mathrm{e}-03$ & $1.38 \mathrm{e}-02$ \\
$1 / 32$ & $4.79 \mathrm{e}-03$ & $5.84 \mathrm{e}-03$ & $4.52 \mathrm{e}-03$ & $2.16 \mathrm{e}-03$ & $3.49 \mathrm{e}-03$ \\
$1 / 64$ & $1.21 \mathrm{e}-03$ & $1.48 \mathrm{e}-03$ & $1.13 \mathrm{e}-03$ & $5.43 \mathrm{e}-04$ & $8.79 \mathrm{e}-04$ \\
$1 / 128$ & $3.64 \mathrm{e}-04$ & $4.31 \mathrm{e}-04$ & $2.88 \mathrm{e}-04$ & $1.49 \mathrm{e}-04$ & $2.47 \mathrm{e}-04$ \\
\hline \hline$O\left(h^{r}\right) r=$ & 1.9025 & 1.8994 & 2.0213 & 1.9454 & 1.9418 \\
\hline \hline
\end{tabular}

Next, we consider this heat problem with a mixed boundary condition

$$
\left\{\begin{aligned}
u=g & \text { on } \partial \Omega_{1}, \\
a \nabla u \cdot n+u=0, & \text { on } \partial \Omega_{2},
\end{aligned}\right.
$$

where $\partial \Omega_{1} \cap \partial \Omega_{2}=\emptyset$, and $\partial \Omega_{1} \cup \partial \Omega_{2}=\partial \Omega$. The exact solution is set to be $u=\sin \left(2 \pi\left(t^{2}+\right.\right.$ 1) $+\pi / 2) \sin (\pi y) e^{-x}$, where $\partial \Omega_{2}$ is the boundary segment $x=1$ and $\partial \Omega_{1}$ is the union of all other boundary segments. For the mixed boundary data type of initial boundary value problem, we also achieved the optimal convergence rates of the error in all norms as shown in Table 3 and Table 4.

Example 2. For the second example, we consider the parabolic problem (37) of full tensor with Dirichlet boundary condition and coefficient matrix $a=\left[\begin{array}{cc}x^{2}+y^{2}+1 & x y \\ x y & x^{2}+y^{2}+1\end{array}\right]$, which 
TABLE 3. Convergence rate for heat equation with Robin boundary condition with $k=h$

\begin{tabular}{||c||c||c||c||c||c||}
\hline \hline$h$ & $\left\|e_{h}\right\|_{\{\infty, T\}}$ & $\left\|e_{h}\right\|_{\{\infty, \partial T\}}$ & $\left\|\nabla_{d} e_{h}\right\|$ & $\left\|e_{h}\right\|_{\left\{L^{2}, T\right\}}$ & $\left\|e_{h}\right\|_{\left\{L^{2}, \partial T\right\}}$ \\
\hline \hline $1 / 8$ & $1.65 \mathrm{e}-01$ & $1.72 \mathrm{e}-01$ & $1.80 \mathrm{e}-01$ & $9.86 \mathrm{e}-02$ & $1.83 \mathrm{e}-01$ \\
$1 / 16$ & $1.00 \mathrm{e}-01$ & $1.02 \mathrm{e}-01$ & $8.55 \mathrm{e}-02$ & $5.86 \mathrm{e}-02$ & $1.09 \mathrm{e}-01$ \\
$1 / 32$ & $5.54 \mathrm{e}-02$ & $5.59 \mathrm{e}-02$ & $4.01 \mathrm{e}-02$ & $3.22 \mathrm{e}-02$ & $5.95 \mathrm{e}-02$ \\
$1 / 64$ & $2.92 \mathrm{e}-02$ & $2.93 \mathrm{e}-02$ & $1.91 \mathrm{e}-02$ & $1.69 \mathrm{e}-02$ & $3.13 \mathrm{e}-02$ \\
$1 / 128$ & $1.50 \mathrm{e}-02$ & $1.50 \mathrm{e}-02$ & $9.24 \mathrm{e}-03$ & $8.67 \mathrm{e}-03$ & $1.60 \mathrm{e}-02$ \\
\hline \hline$O\left(h^{r}\right) r=$ & 0.8656 & 0.8793 & 1.0713 & 0.8767 & 0.8789 \\
\hline \hline
\end{tabular}

TABLE 4. Convergence rate for heat equation with Robin boundary condition with $k=h^{2}$

\begin{tabular}{||c||c||c||c||c||c||}
\hline \hline$h$ & $\left\|e_{h}\right\|_{\{\infty, T\}}$ & $\left\|e_{h}\right\|_{\{\infty, \partial T\}}$ & $\left\|\nabla_{d} e_{h}\right\|$ & $\left\|e_{h}\right\|_{\left\{L^{2}, T\right\}}$ & $\left\|e_{h}\right\|_{\left\{L^{2}, \partial T\right\}}$ \\
\hline \hline $1 / 8$ & $3.18 \mathrm{e}-02$ & $3.90 \mathrm{e}-02$ & $2.61 \mathrm{e}-02$ & $1.88 \mathrm{e}-02$ & $3.57 \mathrm{e}-02$ \\
$1 / 16$ & $8.29 \mathrm{e}-03$ & $1.01 \mathrm{e}-03$ & $6.28 \mathrm{e}-03$ & $4.87 \mathrm{e}-03$ & $9.22 \mathrm{e}-03$ \\
$1 / 32$ & $2.10 \mathrm{e}-03$ & $2.54 \mathrm{e}-03$ & $1.55 \mathrm{e}-03$ & $1.23 \mathrm{e}-03$ & $2.32 \mathrm{e}-03$ \\
$1 / 64$ & $5.24 \mathrm{e}-04$ & $6.34 \mathrm{e}-04$ & $3.88 \mathrm{e}-04$ & $3.08 \mathrm{e}-04$ & $5.83 \mathrm{e}-04$ \\
$1 / 128$ & $1.39 \mathrm{e}-04$ & $1.62 \mathrm{e}-04$ & $1.06 \mathrm{e}-04$ & $8.79 \mathrm{e}-05$ & $1.65 \mathrm{e}-04$ \\
\hline \hline$O\left(h^{r}\right) r=$ & 1.9591 & 1.9785 & 1.9875 & 1.9352 & 1.9383 \\
\hline \hline
\end{tabular}

is symmetric and positive definite, and $f, g$ and $\psi$ are determined by setting the exact solution $u=\sin \left(2 \pi\left(t^{2}+1\right)+\pi / 2\right) \sin (2 \pi x+\pi / 2) \sin (2 \pi y+\pi / 2)$. The results are shown in Table 5 and Table 6 , which confirm the theoretical rates of convergence in $L^{2}$. For the discrete $H^{1}$ norm, the numerical convergence is of order $\mathcal{O}\left(h^{2}\right)$ which is one order higher than the theoretical prediction. We believe that this suggests a superconvergence between the weak Galerkin finite element approximation and the $L^{2}$ projection of the exact solution. Interested readers are encouraged to conduct a study on the superconvergence phenomena.

TABle 5. Convergence rate for parabolic problem with inhomogeneous Dirichlet boundary condition with $k=h$

\begin{tabular}{||c||c||c||c||c||c||}
\hline \hline$h$ & $\left\|e_{h}\right\|_{\{\infty, T\}}$ & $\left\|e_{h}\right\|_{\{\infty, \partial T\}}$ & $\left\|\nabla_{d} e_{h}\right\|$ & $\left\|e_{h}\right\|_{\left\{L^{2}, T\right\}}$ & $\left\|e_{h}\right\|_{\left\{L^{2}, \partial T\right\}}$ \\
\hline \hline $1 / 8$ & $1.21 \mathrm{E}-01$ & $1.38 \mathrm{E}-01$ & $3.57 \mathrm{E}-01$ & $4.64 \mathrm{E}-02$ & $8.14 \mathrm{E}-02$ \\
$1 / 16$ & $5.64 \mathrm{E}-02$ & $6.10 \mathrm{E}-02$ & $1.23 \mathrm{E}-01$ & $1.87 \mathrm{E}-02$ & $3.39 \mathrm{E}-02$ \\
$1 / 32$ & $2.67 \mathrm{E}-02$ & $2.81 \mathrm{E}-02$ & $4.34 \mathrm{E}-02$ & $8.35 \mathrm{E}-03$ & $1.54 \mathrm{E}-02$ \\
$1 / 64$ & $1.29 \mathrm{E}-02$ & $1.33 \mathrm{E}-02$ & $1.55 \mathrm{E}-02$ & $3.95 \mathrm{E}-03$ & $7.29 \mathrm{E}-03$ \\
$1 / 128$ & $6.33 \mathrm{E}-03$ & $6.42 \mathrm{E}-03$ & $5.61 \mathrm{E}-03$ & $1.92 \mathrm{E}-03$ & $3.55 \mathrm{E}-03$ \\
\hline \hline$O\left(h^{r}\right) r=$ & 1.0654 & 1.1076 & 1.4978 & 1.1487 & 1.1301 \\
\hline \hline
\end{tabular}


TABle 6. Convergence rate for parabolic problem with inhomogeneous Dirichlet boundary condition with $k=h^{2}$

\begin{tabular}{||c||c||c||c||c||c||}
\hline \hline$h$ & $\left\|e_{h}\right\|_{\{\infty, T\}}$ & $\left\|e_{h}\right\|_{\{\infty, \partial T\}}$ & $\left\|\nabla_{d} e_{h}\right\|$ & $\left\|e_{h}\right\|_{\left\{L^{2}, T\right\}}$ & $\left\|e_{h}\right\|_{\left\{L^{2}, \partial T\right\}}$ \\
\hline \hline $1 / 8$ & $7.41 \mathrm{E}-02$ & $9.68 \mathrm{E}-02$ & $1.24 \mathrm{E}-01$ & $3.53 \mathrm{E}-02$ & $5.74 \mathrm{E}-02$ \\
$1 / 16$ & $1.92 \mathrm{E}-02$ & $2.56 \mathrm{E}-02$ & $3.00 \mathrm{E}-02$ & $9.14 \mathrm{E}-03$ & $1.47 \mathrm{E}-02$ \\
$1 / 32$ & $4.83 \mathrm{E}-03$ & $6.44 \mathrm{E}-03$ & $7.45 \mathrm{E}-03$ & $2.30 \mathrm{E}-03$ & $3.70 \mathrm{E}-03$ \\
$1 / 64$ & $1.21 \mathrm{E}-03$ & $1.62 \mathrm{E}-03$ & $1.86 \mathrm{E}-03$ & $5.78 \mathrm{E}-04$ & $9.28 \mathrm{E}-04$ \\
$1 / 128$ & $3.35 \mathrm{E}-04$ & $4.34 \mathrm{E}-04$ & $4.67 \mathrm{E}-04$ & $1.53 \mathrm{E}-04$ & $2.48 \mathrm{E}-04$ \\
\hline \hline$O\left(h^{r}\right) r=$ & 1.9474 & 1.9500 & 2.0118 & 1.9637 & 1.9636 \\
\hline \hline
\end{tabular}

\section{REFERENCES}

[1] V. Thomée, Galerkin Finite Element Methods for Parabolic Problems, (Springer Series in Computational Mathematics), Springer-Verlag New York, Inc., Secaucus, NJ, 2006.

[2] K. Eriksson and C. Johnson, "Adaptive Finite Element Methods for Parabolic Problems IV: Nonlinear Problems", SIAM Journal on Numerical Analysis, Vol. 32, No. 6 (1995), pp. 1729-1749.

[3] S. Larsson, V. Thomée and L.B. Wahlbin, "Numerical solution of parabolic integrodifferential equations by the discontinuous Galerkin method", Math. Comp., Vol. 67, (1998), pp. 45-71.

[4] X. Makridakii and I. Babuška, "On the stability of the discontinuous Galerkin method for the heat equation", SIAM J. Num. Anal., Vol. 34, (1997), pp. 389-401.

[5] P. Chatzipantelidis, R. D. Lazarov and V. Thomée, "Error estimates for a finite volume element method for parabolic equations in convex polygonal domains", Numerical Methods for Partial Differential Equation, 20, (2004), pp. 650-674.

[6] M. Yang and J. Liu, "A quadratic finite volume element method for parabolic problems on quadrilateral meshes", IMA Journal of Numerical Analysis, 31, (2011), pp. 1038-1061.

[7] J. Wang and X. Ye, "A weak Galerkin finite element method for elliptic problems", arXiv:1104.2897v1, 2011, Journal of Computational and Applied Mathematics, 241 (2013), pp. 103-115.

[8] P. Raviart and J. Thomas, "A mixed finite element method for second order elliptic problems", Mathematical Aspects of the Finite Element Method, I. Galligani, E. Magenes, eds., Lecture Notes in Math. 606, Springer-Verlag, New York, 1977.

[9] J. Wang and X. Ye, "A Weak Galerkin mixed finite element method for second-order elliptic problems", arXiv: 1202.3655 v2, 2012.

[10] L. Mu, J. Wang, and X. Ye, "Weak Galerkin finite element methods on polytopal meshes", arXiv:1204.3655 v2, 2012.

[11] J. Wang, Y. Wang, and X. Ye, "Unified a posteriori error estimator for finite element methods for the Stokes equations." International J of Numerical Analysis and Modeling, accepted (2012).

[12] L. Mu, J. Wang, and X. Ye, "A new Galerkin finite element method for the Stokes equations", Preprint and private communication.

[13] M.F. Wheeler, "A priori L2 error estimates for Galerkin approximations to parabolic partial differential equations", SIAM Journal on Numerical Analysis, Vol. 10, No. 4 (1973), pp. 723-759.

[14] L. Mu, J. Wang, Y. Wang, and X. Ye, "A computational study of the weak Galerkin method for second-order elliptic equations", Numerical Algorithms, DOI: 10.1007/s11075-012-9651-1, 2012.

Department of Mathematics, Towson University, Towson, MD 22152

E-mail address: qli@towson.edu

Division of Mathematical Sciences, National Science Foundation, Arlington, VA 22230

E-mail address: jwang@nsf.gov 